\title{
Evaluation of Map Signs for Evacuation Purposes
}

\author{
Zdeněk Stachoň $^{\mathrm{a},}$, , Petr Kubíček ${ }^{\mathrm{a}}$, Hana Švedováa ${ }^{\mathrm{a}}$, Jie Shen ${ }^{\mathrm{b}, \mathrm{c}, \mathrm{d}}$, Xinqian Wu ${ }^{\mathrm{b}, \mathrm{c}, \mathrm{d}}$, Milan Konečnýa \\ ${ }^{a}$ Department of Geography, Faculty of Science, Masaryk University, Brno, Czech Republic; zstachon@ geogr.muni.cz.(Z.S.), \\ kubicek@geogr.muni.cz(P.K.),451557@mail.muni.cz(H.S.), konecny@geogr.muni.cz.(M.K.) \\ ${ }^{b}$ Jiangsu Center for Collaborative Innovation in Geographical Information Resource Development and Application, Nanjing \\ 210023, China; \\ ${ }^{c}$ School of Geography Science, Nanjing Normal University, Nanjing 210023, China \\ ${ }^{d}$ Key Laboratory of Virtual Geographic Environment (Nanjing Normal University), Ministry of Education, Nanjing 210023, China; \\ 446196400@qq.com (X.W.), shenjie@njnu.edu.cn (J.S.)
}

* Corresponding author

Keywords: Evacuation map, Cartographic visualization, Evaluation

\begin{abstract}
:
An increasing number of population brings increasing vulnerability of the society to different disasters and emergencies. The maps provide spatial information, which is useful on different levels of decision making during emergencies from strategic planning to single person decision making. To fit the user needs maps can be optimized for a particular user, user group, available technology or external conditions.

Evacuation is a crucial part of the process dealing with most of the emergencies. In case of building the standard ISO 23601:2009, Safety identification - Escape and evacuation plan signs was developed to provide the guidelines for the design, materials, installation and other attributes of the evacuation plans. It is mostly based on the ISO 7010:2003, Graphical symbols - Safety colours and safety signs - Safety signs that are widely used in the buildings all around the world. The symbols were designed with the purpose to attract user attention under any circumstances and across the different cultural background. It can be a disadvantage in case of cartographic visualization, while the symbols on the map are not easy to identify the anchor point, increase the graphics clutter of the map (see figure 1).
\end{abstract}

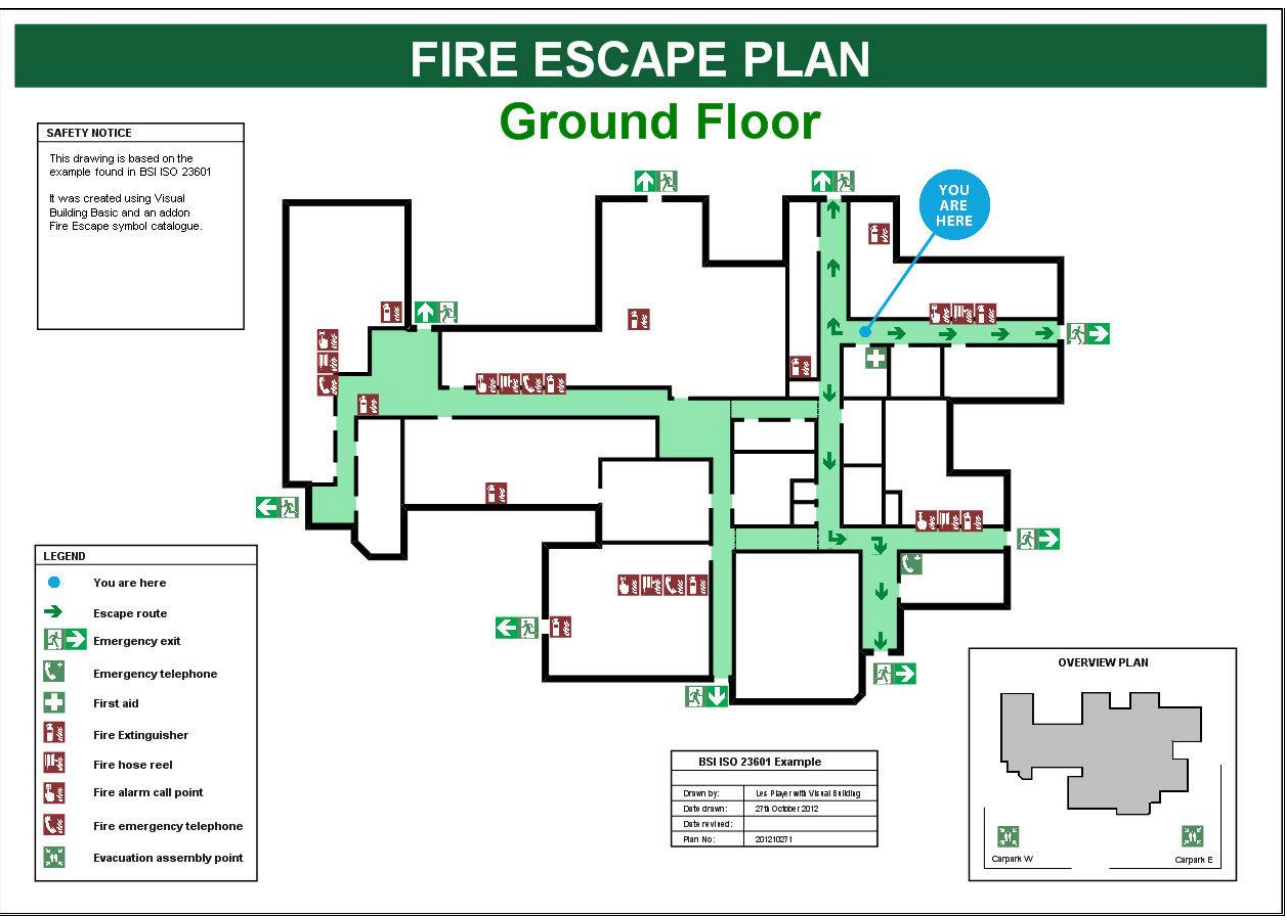

Figure 1. Example of ISO 23601 Escape and Evacuation Plan.

In our research, we have decided to focus on the evaluation of user's abilities to use and understand of designed evacuation signs and plans. As the standard is intended to be used worldwide, we have designed study focused on the comparison of the understanding to the meaning of evacuation symbols in general and also in the form of cartographic visualization. We have designed the user study performed in the first step in Nanjing (China) and in Brno (Czech Republic) in order to verify the cross-cultural universality of evacuation symbols. There were about seventy participants in China and seventy 
participants in the Czech Republic, who performed similar tasks. The tasks consist of separate symbol meaning selection, a separate symbol meaning estimation, identification of a particular symbol on the map and basic interpretation of the map content. The original and modified map signs were used as stimuli in case of cartographic visualization. Results were statistically processed and discussed. The results bring a new perspective on the standardized cartographic visualization for purposes of building evacuation. 